\title{
Xanthinurie Typ 1 bei einer Patientin mit Gelenkschmerzen
}

\section{Eine kombinierte konventionelle und molekulargenetische Ursachensuche}

\author{
Roland Wolfgang Därr, Steffen Lenzner, Thomas Eggermann, Wolfgang Hermann Därr
}

\begin{abstract}
Die Diagnose einer angeborenen Stoffwechselkrankheit kann eine Herausforderung sein: Diese Erkrankungen sind selten, ihr klinisches Bild ist sehr variabel. Bei der hier beschriebenen Patientin führte eine Genmutation zu Gelenkbeschwerden - der einzige auffällige Laborbefund war eine stark erniedrigte Serum-Harnsäure.
\end{abstract}

Unklare Polyarthralgien | Eine 53-jährige Frau stellt sich wegen anhaltender Gelenkschmerzen und einer leichten Schwellung der Zehengrundgelenke I und II beidseits bei ihrem Orthopäden vor. Eine etwa 6 Monate zuvor durchgeführte rheumatologische Untersuchung ist ergebnislos geblieben. Die jetzt veranlassten Laborbestimmungen zeigen als einzige Auffälligkeit einen nicht messbar erniedrigten Wert der SerumHarnsäure. Der Labormediziner empfiehlt, eine IgM-Paraproteinämie als mögliche Ursache auszuschließen. Daher wird die Patientin in die Klinik eingewiesen.

Beschwerden durch Trinken gebessert I Am Untersuchungstag ist die Patientin beschwerdefrei. Abgesehen von dem Gelenkleiden, das seit etwa 2 Jahren besteht, sei sie bislang gesund gewesen. Sie gibt an, mindestens 2,5 Liter täglich zu trinken, seitdem sie bemerkt habe, dass sich darunter ihre Gelenkbeschwerden besserten. Medikamente nehme sie nicht ein. Sie sei verheiratet und habe zwei erwachsene Söhne. Ihre Mutter sei gesund; der Vater sei im Alter von 34 Jahren vermutlich an einem Herzinfarkt verstorben.

Harnsäure stark erniedrigt | Der klinische Untersuchungsbefund und die Abdomensonografie sind unauffällig, ebenso die meisten Laborparameter (Blutsenkung, C-reaktives Protein, Hämatokrit, Blutbild und Differenzialblutbild, Leber-, Nieren- und Schilddrüsenfunktion, Gesamteiweiß, Albumin, Glukose, Elektrolyte, Phosphat, Urinstatus und -sediment).

- Die Serum-Harnsäurekonzentration ist mit $58 \mu \mathrm{mol} / \mathrm{l}(0,97 \mathrm{mg} / \mathrm{dl})$ stark erniedrigt.

- Die fraktionelle Harnsäureausscheidung (FEUA) liegt im Normbereich.

- Die Konzentration von Xanthin im Urin ist 14fach, die von Hypoxanthin 7,5-fach erhöht.

Nach oraler Belastung mit $300 \mathrm{mg}$ Allopurinol steigt die Serum-Oxipurinolkonzentration innerhalb von $90 \mathrm{~min}$ auf $4,0 \mathrm{mg} / \mathrm{l}$ an ( $\triangleright$ Tab. 1).

Molekulargenetische Untersuchung I Die Sequenzierung des Xanthin-Dehydrogenase(XDH)Gens nach Amplifizierung mittels Polymerase-
Kettenreaktion (PCR) und der Vergleich mit der Referenzsequenz NM-000379 zeigt im Exon 8 die homozygote 1-bp-Deletion c.641delC (p.Pro214Glnfs*4). Weitere Mutationen finden sich nicht. Die quantitative Analyse von Exon 8 und den beiden flankierenden Exons 7 und 9 des XDH-Gens mittels Echtzeit-PCR (RT-PCR) ergibt keinen Anhalt für eine Deletion dieses Genabschnitts.

Familienuntersuchung I Bei der Mutter und den beiden Söhnen der Patientin weisen wir die heterozygote Mutation c.641delC im XDH-Gen nach. Ein Halbbruder und eine Halbschwester des verstorbenen Vaters sind keine Anlageträger. Die Serum-Harnsäurekonzentration ist bei allen untersuchten Familienmitgliedern normal. Es besteht kein Hinweis auf elterliche Konsanguinität ( Abb. 1).

Diagnose I Aufgrund dieser Untersuchungsergebnisse stellen wir die Diagnose einer Xanthinurie Typ 1. Es handelt sich um die erste Dokumentation einer nicht verwandschaftsbedingten Homozygotie für die Mutation c.641delC im XDH-Gen.

Therapieempfehlung | Wir klären die Patientin über ihre Erkrankung auf und raten ihr, die gesteigerte tägliche Trinkmenge beizubehalten und zusätzlich auf eine purinarme Kost zu achten. Die Patientin ist seither beschwerdefrei geblieben.

\section{Diskussion}

Störungen im Purinstoffwechsel | Angeborenen Stoffwechselkrankheiten liegt eine genetisch bedingte Störung der Proteinsynthese zugrunde. Ihr Erbgang ist überwiegend autosomal rezessiv [17]. Das klinische Bild und der Verlauf sind hoch variabel - die Diagnose kann deshalb eine Herausforderung sein $[12,13,19,24]$. Im Stoffwechsel der Purinbasen sind drei angeborene Störungen bekannt, die den letzten Schritt der Harnsäurebildung betreffen. Alle führen zu einer 


\begin{tabular}{|c|c|c|c|}
\hline & Parameter & Befund & Normalbereich \\
\hline \multirow[t]{8}{*}{ Blut } & Kreatinin & $76,8 \mu \mathrm{mol} / \mathrm{I}$ & 53-97 $\mu \mathrm{mol} / \mathrm{l}$ \\
\hline & Harnsäure & $58,0 \mu \mathrm{mol} / \mathrm{l}$ & $137-363 \mu \mathrm{mol} / \mathrm{I}$ \\
\hline & Gesamteiweiß & $78,3 \mathrm{~g} / 1$ & $66-87 \mathrm{~g} / 1$ \\
\hline & Albumin & $48,8 \mathrm{~g} / 1$ & $35-52 \mathrm{~g} / 1$ \\
\hline & Calcium & $2,46 \mathrm{mmol} / \mathrm{l}$ & $2,15-2,57 \mathrm{mmol} / \mathrm{I}$ \\
\hline & Phosphat & $1,14 \mathrm{mmol} / \mathrm{l}$ & $0,87-1,45 \mathrm{mmol} / \mathrm{I}$ \\
\hline & TSH & $1,50 \mathrm{mlU} / \mathrm{l}$ & $0,40-4,00 \mathrm{mlU} / \mathrm{I}$ \\
\hline & Oxipurinol & $4,00 * * \mathrm{mg} / \mathrm{I}$ & $5,80+1,5 \mathrm{mg} / \mathrm{I}^{*}$ \\
\hline \multirow[t]{7}{*}{ Urin } & Glukose & negativ & negativ \\
\hline & $\mathrm{pH}$ & 7,0 & $4,8-7,5$ \\
\hline & Kreatinin & $14334 \mu \mathrm{mol} / 24 \mathrm{~h}$ & $6600-13900 \mu \mathrm{mol} / 24 \mathrm{~h}$ \\
\hline & Harnsäure & $131 \mu \mathrm{mol} / \mathrm{l}$ & $800-4000 \mu \mathrm{mol} / \mathrm{I}$ \\
\hline & Xanthin & $229,2 \mathrm{mg} / 24 \mathrm{~h}$ & $3,9-16,7 \mathrm{mg} / 24 \mathrm{~h}$ \\
\hline & Hypoxanthin & $75,1 \mathrm{mg} / 24 \mathrm{~h}$ & $3,0-10 \mathrm{mg} / 24 \mathrm{~h}$ \\
\hline & FEUA*** & $3,9 \%$ & $<10 \%$ \\
\hline
\end{tabular}

Tab. 1 Laboruntersuchungen. *Peak-Plasma-Konzentration $2 \mathrm{~h}$ nach oraler Belastung mit 300 mg Allopurinol [3, 34]; ${ }^{* *}$ Wert 90 min nach Allopurinol-Belastung; ${ }^{* * *}$ fraktionelle Harnsäureausscheidung [4]

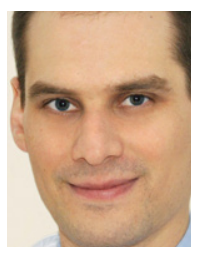

Dr. med. Roland Därr ist Facharzt für Innere Medizin, Diabetologie und Endokrinologie; zur Zeit Stipendiat der Deutschen Forschungsgemeinschaft am $\mathrm{NIH} / \mathrm{NICHD}$, Bereich Neuroendokrinologie, in Bethesda, MD, USA. roland_daerr@web.de

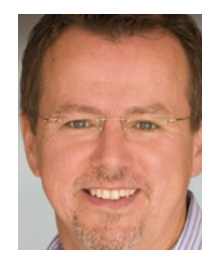

Dr. rer. nat. Steffen Lenzner ist Abteilungsleiter

(Teilbereich Molekulargenetik), Institut für medizinische Diagnostik GmbH, Ingelheim. steffen.lenzner@bioscientia. de vermehrten Ausscheidung von Xanthin und Hypoxanthin im Urin. Unterschieden werden:

1. Xanthinurie Typ 1: Funktionsverlust der XDH infolge eines Defekts des XDH-Gens

2. Xanthinurie Typ 2: Funktionsverlust der XDH und Aldehyd-Oxidase (AO) infolge eines Defekts des Molybdän-Cofaktor(MoCo)-Sulfurase-Gens

3. MoCo-Defizienz: Funktionsverlust der $\mathrm{XDH}$, AO und Sulfitoxidase (SO) infolge einer Synthesestörung von $\operatorname{MoCo}([9,10,16,25,27]$.

Erscheinungsbild | Die Xanthinurie Typ 1 und 2 unterscheiden sich nicht in ihrem klinischen Erscheinungsbild. Beide Erkrankungen können durch

- Arthralgien,

- Myalgien und

- Bildung von Xanthinsteinen

auffällig werden und als schwerwiegendste Komplikation ein Nierenversagen verursachen [28]. Die sehr seltene MoCo-Defizienz äußert sich in der Regel kurz nach der Geburt mit einer schweren neurologischen Symptomatik und führt aufgrund einer progredienten Hirnschädigung häufig im ersten Lebensjahr zum Tod [33].

Differenzialdiagnose Hypourikämie | Organerkrankungen oder Medikamente, die zu einer verminderten Synthese oder einer vermehrten renalen Ausscheidung von Harnsäure führen, gehen mit einer mäßig erniedrigten SerumHarnsäurekonzentration ( $<2,0 \mathrm{mg} / \mathrm{dl}$ bzw. $<120$ $\mu \mathrm{mol} / \mathrm{l}$ ) einher (sekundäre Hypourikämie) [1,
4]. Stark erniedrigte Serum-Harnsäurekonzentrationen $(<1,0 \mathrm{mg} / \mathrm{dl}$ bzw. $<60 \mu \mathrm{mol} / \mathrm{l})$ müssen dagegen immer an eine Xanthinurie oder eine hereditäre renale Hypourikämie (RHUC1 und 2) denken lassen $[7,9,20]$. Bei RHUC1 und 2 besteht ein erhebliches Risiko nach anstrengender körperlicher Belastung ein akutes Nierenversagen zu erleiden $[11,18,31]$. Dieser Verdacht sollte rasch differenzialdiagnostisch geklärt werden - das ist über die Bestimmung der FEUA leicht möglich: Bei RHUC1 und 2 ist die FEUA signifikant erhöht, bei einer Xanthinurie normal [15, 22, 30].

Pseudohypourikämie | Eine ungewöhnliche Ursache nicht messbar erniedrigter Werte der SerumHarnsäure sind Fehlbestimmungen aufgrund einer IgM-Paraproteinämie. Die Häufigkeit der Pseudohypourikämie ist nicht bekannt. Bezeichnenderweise ist sie testabhängig, nicht vorhersehbar und schlecht reproduzierbar [5].

- Eine stark erniedrigte Serum-Harnsäurekonzentration sollte deswegen immer auch an eine IgM-Paraproteinämie denken lassen,

- eine gezielte Suche jedoch erst nach Ausschluss einer Xanthinurie und RHUC erfolgen.

Diagnostik bei Xanthinurie | Um zwischen einer Xanthinurie Typ 1 und 2 unterscheiden zu können, führten wir bei unserer Patientin einen Allopurinol-Belastungstest durch. Er beruht darauf, dass die Aldehyd-Oxidase (AO) bei einer Xanthinurie Typ 1 funktionell normal aktiv ist und Allopurinol in Oxipurinol umwandeln kann [26]. In letzter Zeit ist es auch möglich geworden

- spezifische endogene Metabolite der AO im Urin zu quantifizieren und

- die katalytische Aktivität der AO direkt zu bestimmen [23].

Die Diagnose der Xanthinurien wird dadurch vereinfacht und die Exposition mit dem potenziell allergenen Allopurinol vermieden $[21,35]$.

Lokale Häufung des Genotyps I Die Analyse des Genotyps ergab bei der Patientin eine Xanthinurie Typ 1 infolge einer Homozygotie für die 1-bp Deletion c.641delC (p.Pro214Glnfs*4) im Exon 8 des XDH-Gens . Damit im Einklang steht die Heterozygotie bei ihrer Mutter und ihren Söhnen. Die Mutation c.641delC wurde erstmals im Jahr 2010 im compound-heterozygoten Zustand bei einer 78-jährigen polnischen Patientin mit Xanthinurie Typ 1 beschrieben [14], seither noch dreimal bei nicht verwandten tschechischen Patienten ebenfalls als Compound-Heterozygotie $[21,29]$.

Angesichts 18 gegenwärtig bekannter Funktionsverlustmutationen des XDH-Gens und etwa 150 dokumentierter Fälle einer Xanthinurie weltweit $[6,9,28]$ ist dies eine bemerkenswerte Häufung in den beiden Ländern. 


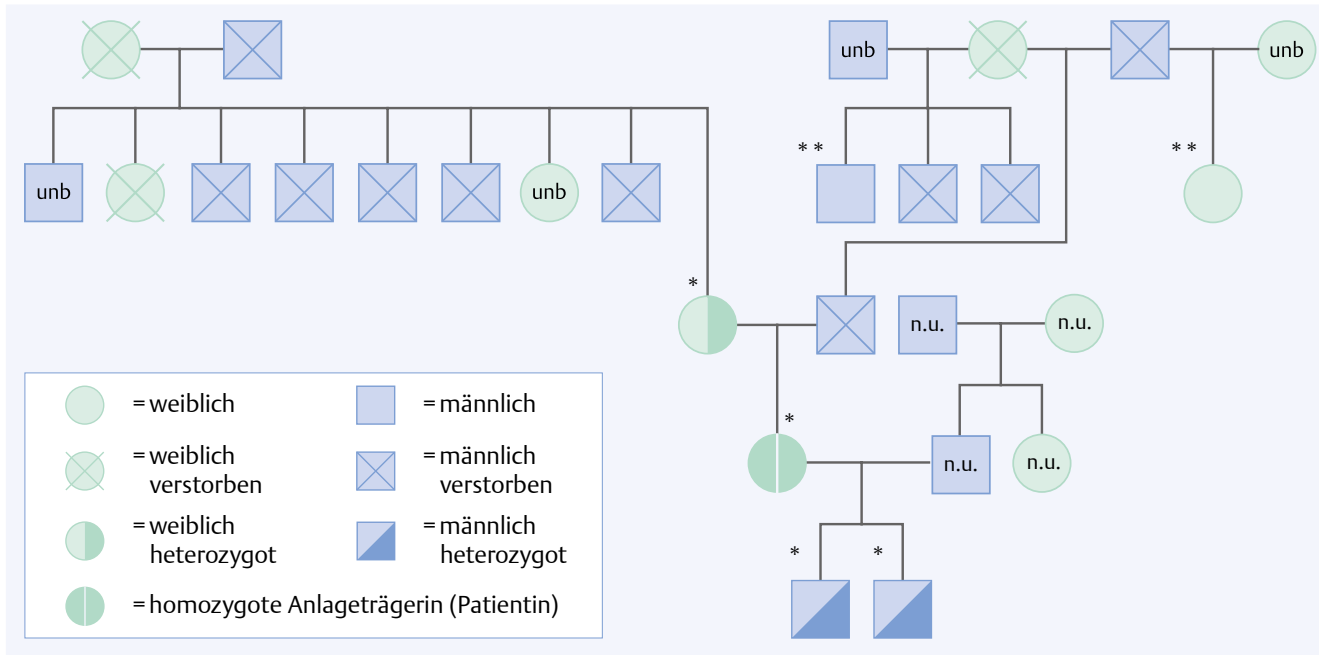

Abb. 1 Stammbaum der Patientin. * Familienmitglieder mit der Mutation c.641delC des XDH Gens **Familienmitglieder ohne diese Mutation; unb. = unbekannt; n.u. = nicht untersucht.

Sie deutet auf eine lokal erhöhte Prävalenz der Mutation c.641delC hin und könnte den homozygoten Genotyp der Patientin erklären, die im Länderdreieck Sachsen-Tschechien-Polen wohnt. Eine Verwandtenehe war aufgrund der Familienuntersuchung auszuschließen.

Prognose und Therapie I Die Prognose der Xanthinurie Typ 1 und 2 ist gut. Selten entwickeln sich ernste Komplikationen wie

- Nierenkoliken,

- rezidivierende Harnwegsinfekte,

- ein akutes Nierenversagen

- oder eine terminale Niereninsuffizienz.

Die Urolithiasis ist eine typische Komplikation im Kindesalter. Myalgien und Arthralgien aufgrund der Ablagerung von Xanthinkristallen beobachtet man vorwiegend im mittleren und höheren Erwachsenenalter [2 ,8, 14, 21, 32]. Bei weitem am häufigsten wird die Diagnose jedoch bei völlig asymptomatischen Geschwistern eines betroffenen Patienten oder aufgrund einer zufälligen Bestimmung der Serum-Harnsäure gestellt [28]. Eine kausale Therapie gibt es nicht. Die Behandlung beschränkt sich auf einen erhöhten Flüssigkeitsumsatz und eine purinarme Kost.

\section{Konsequenz für Klinik und Praxis}

- Stark erniedrigte Harnsäurewerte im Serum sind Kennzeichen der Xanthinurie und der hereditären renalen Hypourikämie.

- Eine Pseudohypourikämie ist erst nach Ausschluss dieser Erkrankungen in Erwägung zu ziehen.

- Die Behandlung einer Xanthinurie ist symptomatisch. Sie beschränkt sich auf einen erhöhten Flüssigkeitsumsatz und eine purinarme Kost.

\section{Literatur}

1 Bairaktari ET, Kakafika Al, Pritsivelis N et al. Hypouricemia in individuals admitted to an inpatient hospital-based facility. Am J Kidney Dis 2003; 41: 1225-1232

2 Bradbury MG, Henderson M, Brocklebank JT et al. Acute renal failure due to xanthine stones. Pediatr Nephrol 1995; 9: 476-477

3 Breithaupt B, Tittel M. Kinetics of allopurinol after single intravenous and oral doses. Noninteraction with benzbromarone and hydrochlorothiazide. Eur J Clin Pharmacol 1982; 22: 77-84

4 Bugdayci G, Balaban Y, Sahin O. Causes of hypouricemia among outpatients. Lab Medicine 2008; 39: 550-552

5 Dalal BI, Brigden ML. Factitious biochemical measurements resulting from hematologic conditions. Am J Clin Pathol 2009; 131: 195-204

6 Eggermann T, Spengler S, Denecke B et al. Multi-exon deletion in the XDH gene as a cause of classical xanthinuria. Clin Nephrol 2013; 79: 78-80

7 Enomoto A, Kimura H, Chairoungdua A et al. Molecular identification of a renal urate anion exchanger that regulates blood urate levels. Nature 2002; 417: 447-452

8 Gargah T, Essid A, Labassi A et al. Xanthine urolithiasis. Saudi J Kidney Dis Transpl 2010; 21: 328-331

9 Ichida K, Amaya Y, Okamoto K et al. Mutations associated with functional disorder of xanthine oxidoreductase and hereditary xanthinuria in humans. Int J Mol Sci 2012; 13: 15475-15495

10 Ichida K, Matsumura T, Sakuma R et al. Mutation of human molybdenum cofactor sulfurase gene is responsible for classical xanthinuria type II. Biochem Biophys Res Commun 2001; 282: 1194-1200

11 Jeannin G, Chiarelli N, Gaggiotti M et al. Recurrent exercise-induced acute renal failure in a young Pakistani man with severe renal hypouricemia and SLC2A9 compound heterozygosity. BMC Med Genet 2014; 15: 3

12 Jurecka $A$. Inborn errors of purine and pyrimidine metabolism. J Inherit Metab Dis 2009; 32: 247-263

13 Jurecka A. [Inborn errors of purine and pyrimidyne metabolism]. Postepy Biochem 2011; 57: 172-182

Vollständiges Literaturverzeichnis unter http://dx.doi.org/10.1055/s-0041-106053

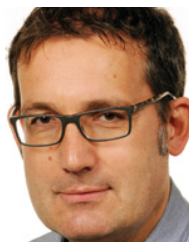

Prof. Dr. rer. nat. Thomas Eggermann ist Laborleiter am Institut für Humangenetik der RWTH Aachen. teggermann@ukaachen.de

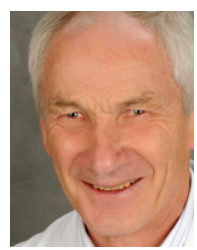

Prof. Dr. med. Wolfgang Därr

ist ehem. Direktor der Klinik für Innere Medizin - Nephrologie, Vivantes Klinikum im Friedrichshain, Berlin; zur Zeit nephrologischer Konsiliararzt am Klinikum Döbeln. wolfgang-daerr@t-online.de

Interessenkonflikt Die Autoren geben an, dass kein Interessenkonflikt besteht.

DOI 10.1055/s-0041-106053

Dtsch Med Wochenschr 2016; 141: 571-573

(c) Georg Thieme Verlag KG . Stuttgart · New York . ISSN 0012-0472 
14 Jurecka A, Stiburkova B, Krijt J et al. Xanthine dehydrogenase deficiency with novel sequence variations presenting as rheumatoid arthritis in a 78-year-old patient. J Inherit Metab Dis 2010; 33 (Suppl 3): S21-24

15 Kikuchi Y, Koga H, Yasutomo Y et al. Patients with renal hypouricemia with exercise-induced acute renal failure and chronic renal dysfunction. Clin Nephrol 2000; 53: 467-472

16 Klein JM, Schwarz G. Cofactor-dependent maturation of mammalian sulfite oxidase links two mitochondrial import pathways. J Cell Sci 2012; 125: 4876-4885

17 Lam STS. Genetic aspects of inherited metabolic diseases. Hong Kong Med J 1996; 2: 285-291

18 Li Z, Ding H, Chen C et al. Novel URAT1 mutations caused acute renal failure after exercise in two Chinese families with renal hypouricemia. Gene 2013; 512: 97-101

19 Mak CM, Lee HC, Chan AY, et al. Inborn errors of metabolism and expanded newborn screening: review and update. Crit Rev Clin Lab Sci 2013; 50: 142-162

20 Matsuo H, Chiba T, Nagamori S et al. Mutations in glucose transporter 9 gene SLC2A9 cause renal hypouricemia. Am J Hum Genet 2008; 83: 744-751

21 Mraz M, Hurba O, Bartl J, et al. Modern diagnostic approach to hereditary xanthinuria. Urolithiasis 2015; 43: 61-67

22 Ohta T, Sakano T, Igarashi T et al. Exercise-induced acute renal failure associated with renal hypouricaemia: results of a questionnaire-based survey in Japan. Nephrol Dial Transplant 2004; 19: 14471453

23 Peretz H, Watson D, Blackburn G et al. Urine metabolomics reveals novel physiologic functions of human aldehyde oxidase and provides biomarkers for typing xanthinuria. Metabolomics 2012; 8: 951-959

24 Raghuveer TS, Garg U, Graf WD. Inborn errors of metabolism in infancy and early childhood: an update. Am Fam Physician 2006; 73: 1981-1990

25 Reiss J, Johnson JL. Mutations in the molybdenum cofactor biosynthetic genes MOCS1, MOCS2, and GEPH. Hum Mutat 2003; 21: 569-576
26 Reiter S, Simmonds HA, Zollner $N$ et al. Demonstration of a combined deficiency of xanthine oxidase and aldehyde oxidase in xanthinuric patients not forming oxipurinol. Clin Chim Acta 1990; 187: 221-234

27 Schwarz G, Mendel RR, Ribbe MW. Molybdenum cofactors, enzymes and pathways. Nature 2009; 460: 839-847

$28 \mathrm{Simmonds} \mathrm{HA}$. Hereditary xanthinuria. Orphanet Encyclopedia 2003. http://www.orpha.net/data/ patho/GB/uk-XDH.pdf

29 Stiburkova B, Krijt J, Vyletal P et al. Novel mutations in xanthine dehydrogenase / oxidase cause severe hypouricemia: biochemical and molecular genetic analysis in two Czech families with xanthinuria type I. Clin Chim Acta 2012; 413: 93-99

30 Stiburkova B, Taylor J, Marinaki AM et al. Acute kidney injury in two children caused by renal hypouricaemia type 2. Pediatr Nephrol 2012; 27: 1411-1415

31 Takahashi T, Tsuchida S, Oyamada T et al. Recurrent URAT1 gene mutations and prevalence of renal hypouricemia in Japanese. Pediatr Nephrol 2005; 20: $576-578$

32 Thomas N, Stephen DC, Abraham B et al. Xanthinuria--an unusual cause for renal stone disease. J Assoc Physicians India 1996; 44: 203-204

33 Veldman A, Santamaria-Araujo JA, Sollazzo S et al. Successful treatment of molybdenum cofactor deficiency type A with cPMP. Pediatrics 2010; 125 : e1249-1254

34 Walter-Sack I, Gröbner W, Zöllner N. [Time course of oxipurinol levels in plasma following single dose and chronic administration of allopurinol in different pharmaceutical preparations (author's transl)]. Arzneimittelforschung 1979; 29: 839-842

35 Yun J, Mattsson J, Schnyder K et al. Allopurinol hypersensitivity is primarily mediated by dosedependent oxypurinol-specific T cell response. Clin Exp Allergy 2013; 43: 1246-1255 